\title{
Using tactile pressure sensors to measure dynamic earth pressures around dual-row walls
}

\author{
SSC Madabhushi, SK Haigh
}

\begin{abstract}
Following the destructive 2011 Tohoku earthquake and tsunami, dual-row retaining walls are being employed in Japan to protect coastal areas from these natural hazards. Dynamic centrifuge tests are conducted to investigate the system behaviour, in particular the earth pressures generated. Highfrequency tactile pressure sensors can measure the dynamic earth pressures. By directly measuring the stresses generated during earthquake events invaluable insight can be gained into the dynamic behaviour. However, the processing and interpretation of the raw output can be challenging. This paper describes a processing scheme that can be used to reduce the apparent scatter in the data. This is applied to the earth pressure data collected using the system developed by Tekscan. The calibration and sensitivity correction process is detailed, with attention to the limitations of the assumptions made in the proposed method. The variation of the processed earth pressures with time is considered to better understand the wall behaviour. The distribution along the wall is considered at several instants to gain further insight. To help verify the earth pressure measurements, equilibrium-based methods are used to investigate the consistency between the recorded earth pressures and the outputs from other instruments that directly measure the wall response.
\end{abstract}

\section{Introduction}

The devastation to coastal communities caused by earthquake and tsunami loadings in recent times has made clear the responsibility on the modern engineer to protect populations from these natural hazards. Dual-row retaining walls are currently being constructed by the piling company Giken Ltd to protect coastal areas in Japan. The structure is illustrated conceptually in Figure 1. The two rows of sheet piles are implanted into existing levees with the pile heads typically connected by tie rods. This allows tall structures to be produced that have a relatively small lateral extent.

The concept of two-tied parallel rows of piles was implemented to achieve a large lateral resistance of the retaining wall system to both earthquake and tsunami loadings. The destructive tsunamis in recent times have been triggered by a preceding earthquake. Thus, the evaluation of the performance of these structures under earthquake loading is a crucial goal.

The seismic design of such structures often relies on simplistic predictions of the limiting earth pressure distributions, such as those described by Mononobe and Matsuo (1929) or Okabe (1924). The simplifying assumptions built into these equations have been discussed in previous literature (Steedman and Zeng, 1990). Further, the applicability of these predictions to the novel case of dualrow retaining walls has not been well established. Ultimately, the earth pressures that develop will depend on the strains that are induced in the soil, which will be related to the manner in which the structure responds to lateral loading. Although accurate prediction of the static or dynamic earth pressures represent a significant academic challenge, the need to check the simplified bounds used for industrial applications may be motivated by both increased safety and efficiency. 
In Madabhushi and Haigh (2016b), the dynamic earth pressures developed during seismic tests on centrifuge models of dual-row structures with stiff walls were detailed, following the calibration and processing of the raw output to the final earth pressure distributions. In an extension to that paper, the same processing technique is adopted for dual-row structures with relatively flexible walls, the flexural stiffness having been reduced by a factor of 8 . The processing technique will be considered in more detail in this paper and its ability to predict the more complicated earth pressures resulting from a flexible dual-row system will be reviewed.

\section{Centrifuge Model}

It is well recognised that the behaviour of soil is non-linear even at small strains, and thus it is incumbent on geotechnical physical modellers to ensure that the stresses expected in the field are applied to the model. A geotechnical centrifuge allows this criterion to be met while requiring only small-scale models to be constructed. An additional advantage for investigating the seismic performance of geotechnical structures is that the models can be inexpensively tested to destruction.

In the experiments discussed here, the $10 \mathrm{~m}$ Turner beam centrifuge at the University of Cambridge was utilised, as described by Schofield (1980). The centrifuge applied an acceleration of 60g at a point $1 / 3$ the height of the dual-row retaining wall structure. Madabhushi et al. (1998) explains the mechanical shaker used in these tests.

Figure 2 illustrates a simplified schematic of the centrifuge model cross-section with the key instruments and relevant dimensions. In order to minimise boundary effects from the rigid container walls Duxseal inserts lining the model edges. The degree to which these absorb the stress wave reflections has been reviewed and discussed, by Campbell et al. (1991) or Coe et al. (1985) for example. A dense soil model (Id $\approx 80 \%$ ) of S28 Hostun Sand (Azeiteiro et al., 2017) was created using the automatic sand pourer (Madabhushi et al., 2006) which uses the dry pluviation method to achieve uniform and repeatable relative densities.

Once pouring of the sand reached the base of the walls, the dual-wall system was placed with the pouring continued in and around them. Installing the walls at $1 \mathrm{~g}$ means that the construction process is not well modelled by these centrifuge tests and the static in situ stresses may not reflect the field case. However, during the application of large earthquake loading, the field stress state will be better captured by the model as the soil tends to the limit states in both cases. The instrumentation discussed in this paper will be the tactile pressure sensing Tekscan sheets inside and outside the right-hand wall, piezoelectric accelerometers measuring input acceleration and the full bridge strain gauges measuring the bending strains in the right-hand wall. The details of the prototype wall system being simulated are given in Table 1. Unless stated otherwise the results will be given at prototype scale.

\section{Tactile Pressure Sensors}

The horizontal effective stresses that develop in the soil are a key consideration for many embedded geotechnical structures. The use of tactile pressure sensors to measure the earth pressures represents a minimally invasive option to obtain key measurements for both practical design and verification of analytical models. The system developed by Tekscan is one such option for the small-scale physical models used in a centrifuge. The sensing sheets used in this work are described as ' $5250-25$ psi' sheets; the first number referring to the original sheet dimensions while the second indicates the sensing range at the default sensitivity setting, in this case $25 \mathrm{psi}$ or $172 \mathrm{kPa}$. The maximum and minimum 
sensitivity settings typically reduce and increase this value by three times respectively. For the flexible wall system investigated the more sensitive 25 psi sheets were preferable to the 250 psi sheets used with the stiffer walls in Madabhushi and Haigh (2016b). The sheets were trimmed to the wall height and contained approximately

30 rows and nine columns giving a total of 270 sensing elements, each with an area of $31.23 \mathrm{~mm}$. The strips had a width of $60 \mathrm{~mm}$ and were placed on the wall $50 \mathrm{~mm}$ behind the front edge.

It should be noted that the sheets are expected to be sensitive to the normal force component and thus should not register any shear stresses developed between the wall and soil. To minimise the development of these stresses the regions of the wall without the Tekscan sheets also had a plastic cover to help maintain consistent interfacial properties across the model. The importance of a smooth interface and the effect of shear stress on the Tekscan output is considered further by Palmer et al. (2009).

Paikowsky and Hajduk (1997) is an example of early work concentrating on the application of the Tekscan system to granular materials, and give details of the physical construction and operation of the sensing sheets - that is how a change in conductivity proportional to the applied pressure is translated from an electrical signal to a digital output which varies between 0 and 255. The paper emphasises the need to carefully calibrate the sheets when used for geotechnical applications, and discusses the possible requirement to calibrate each sensing element individually. The importance of calibrating the Tekscan sheets by applying the pressure by way of a granular interface, rather than directly to the sheets is also highlighted. When using a pressure bladder, for example, each sensing element should experience a uniform pressure. However, because grains of sand apply the pressure to the sheets at discrete contact points, each sensing element may not experience a uniform pressure across its area. This can lead to a different average conductivity, and thus output from the sensing element for the equivalent input pressure. The dependence of the calibration factor on the ratio of the grain size or number of contacts and the sensing area for the Tekscan sheets has not been well established, but by applying the calibrating pressure through the same material the uncertainty can be mitigated. Further, the Hostun Sand used in these tests was unlikely to have particle diameters larger than $0.8 \mathrm{~mm}$, which suggests it is likely that multiple grains rest on each sensing element.

One challenge with calibrating the sheets for centrifuge applications is how to recreate the large stresses expected during the centrifuge test, especially given the need to apply the pressure through the granular material being investigated. Ganainy et al. (2014) discuss the possibility of calibrating the sheets in a secondary centrifuge test by placing the sheets horizontally in a simple soil model and linking the known weight of supported soil at different g levels to the Tekscan output. The sheets are then removed and placed vertically in the actual soil model for measuring lateral soil pressures. However, the need to create additional flat soil models at the same relative density as the real test, as well as fly the centrifuge just to calibrate the Tekscan sheets is both labour and cost intensive. The method proposed in Madabhushi and Haigh (2016b) combines calibration at $1 \mathrm{~g}$ with the results from the centrifuge to aim to provide an accurate but quicker way to use the Tekscan sheets in centrifuge models.

\section{Tekscan calibration and sensitivity adjustment}

Figure 3 illustrates the typical raw output from the sheets. In order to convert this output into earth pressures, the calibration procedure initially introduced in Madabhushi and Haigh (2016b) and illustrated in Figure 4 is followed. Any initial preparation for the centrifuge model, such as trimming or laminating the sheets is completed before the calibration commences. The subsequent process is 
separated to achieve two distinct outcomes. The first aspect is conversion of the relative Tekscan output to an absolute scale, which can be achieved by way of an average calibration factor for the entire sheet. However, it was demonstrated that it is important to recognise the differing sensitivities between individual sensing elements on the sheet. This can be realised using data obtained during swing up, as originally detailed in Madabhushi and Haigh (2016b) and as will be discussed in Section 4.2.

\section{Absolute Calibration}

It is important to try establish the reasons that individual sensing elements, which are part of the same Tekscan sheet, may have differing calibration factors. Variations during the production of the sheets could lead to differing responses between the sensing elements. Further, during the life of the sheets, different elements experience different loadings, which may also introduce variations in the calibration factors. However, these sources should not be confused with the potential for uneven distribution of pressures across the sensing elements due to the use of the sheets with a granular material.

Typically, Tekscan sheets can be calibrated by applying known loads over the sheet and recording the total output across all the sensing elements. In Madabhushi and Haigh (2016b), the method of directly applying load between foam-padded pistons was highlighted as a potential area for amelioration. Specifically, concerns over the distribution of the loading imply the use of pressure bladders as an improvement. In order to better understand the impact of using the sheets to measure the earth pressures in sands, the same sheets were calibrated using a pressure bladder in direct contact with the sheets and then through a $50 \mathrm{~mm}$ layer of dense sand, poured using the same automated pluviation technique described in Section 2.

The pressure applied was automatically cycled to increase the volume of data collected during calibration and thus increase its statistical significance. An example of the response to this cycling is illustrated in Figure 5. For the ranges tested, there was no significant variation in the outputs when compared against the moderate number of cycles applied.

Figure 6 presents illustrative results from correlating the individual sensing element output with the applied pressure. In Figure 6(a) the data collected when the pressure bladder was directly applied to a Tekscan sheet is shown. Linear fits were constructed to the data obtained from each sensing element across cycles and the distributions of the gradients and intercepts are also shown in Figure 6(a). For sensing elements where the larger applied pressures saturated the output the fits ignored these points, based on checks of strictly increasing output for increased pressure. The negative intercepts indicate the sensing elements that produce no output below a certain applied pressure.

From Figure $6(\mathrm{~b})$ the variance of gradients is clearly increased by calibrating through a layer of dense sand, although the range is perhaps less affected. The difference between Figures $6(\mathrm{a})$ and $6(\mathrm{~b})$ suggests that despite the sheets being stiffened by the lamination, the pressure applied through point contacts may still have been non-uniform across the sensing element. The distribution of intercepts in Figure $6(\mathrm{~b})$ is predominantly negative. This may be partly attributed to soil arching redistributing the stresses in the sand layer, as described by Michalowski and Park (2003) for example.

Overall, it is evident that between sensing elements there is a variation both in the calibration gradient and intercept, and that using the sheets with a granular medium can exacerbate the difference. This is in agreement with the original findings by Paikowsky and Hajduk (1997).

\section{Relative Sensitivity}


Theoretically, the individual calibration factors obtained from the pressure vessel method may be applied directly to data collected from a centrifuge test. This would automatically account for the differing calibration factors between sensing elements. On the other hand, Madabhushi and Haigh (2016b) suggests obtaining an average calibration factor at $1 \mathrm{~g}$ and correcting for individual sensitivity using data collected from the swing-up stages of a centrifuge test. This is also summarised in Figure 4.

Essentially, the raw output should be recorded as the centrifuge is held at given g levels during swing up. For each sensing element, a linear fit can be constructed linking the raw output value to the $g$ level. Physically, this is based on the fact that as the $\mathrm{g}$ level is increased the soil stress will generally increase linearly, and that the sheet can be taken to respond linearly between certain threshold pressures (Paikowsky and Hajduk, 1997).

By assuming plane strain conditions, the gradients of the linear fits of raw output against $g$ level for each sensing element may be normalised by the mean of the gradients for each row. This produces an estimate of the sensitivity of each sensing element relative to its row. It is important to do this per row as each row of the sheet experiences a different change in pressure as the $\mathrm{g}$ level is increased during swing up. The normalised sensitivities are applied to each sensing element as well as the calibration factor to improve the accuracy of future measurements.

Although obviously useful for tests where individual calibration data is unavailable but swing-up data is, the relative sensitivity method may also prove beneficial for other reasons. The error on an average calibration factor is clearly lower than on individual values, which reduces the onus on the $1 \mathrm{~g}$ calibration set-up. The underlying assumption in the sensitivity normalisation across the sensor columns is that constant lateral earth pressures are mobilised in the plane strain direction of the model. This is considered reasonable for the central portion of the relatively stiff walls the sheets were adhered to. A disadvantage of the method is that obtaining the relative sensitivity from the sheets as they are placed in the model will confound two effects of why the output across the columns at a given height were not constant to begin with. The natural variation in the calibration factor as shown in Figure 6(a) is rightly corrected for. However, differences arising due to the varying grain contacts will also be averaged out. This may be considered reasonable for subsequent measurements if there is no significant grain rearrangement near the sensing sheets, and will be discussed further in Section 4.3.

The broader benefit of the method, which aims to improve the concurrency between the independent columns of the sensing elements is relevant nevertheless (Madabhushi and Haigh, 2016b). By maintaining the multiple measurements of soil pressure distributions across the sheet the confidence in following measurements is increased.

\section{Example Processing}

Figure 7 illustrates the effect of adjusting for the sensitivities using the data collected from swing up. The solid lines in Figure 7 indicate a trimmed mean of the data points, where the extreme $20 \%$ of each row of data is ignored from the average. The comparison between Figures $7(a)$ and $7(b)$, which illustrates the effect of the sensitivity adjustment at a time after one small earthquake and after several larger earthquakes, respectively, is quite revealing. The large reduction in scatter in Figure $7(a)$ is expected; the sensitivity corrections were derived from the data collected during swing up and assuming plane strain conditions. Thus, for constant assumed pressures the natural variability and that arising from the sand grains in the calibration factor are accounted for. However, although the scatter in Figure $7(b)$ is increased following the earthquake shaking, there is still a significant improvement over the raw data. A large amount of grain rearrangement is likely to have occurred 
following several large shaking events and thus the consistency of the data in Figure 7 (b) goes some way to validate the sensitivity correction method employed.

Comparison of the averaged distributions in Figure 7 may appear to suggest the sensitivity correction only leads to modest overall changes in the distributions. However, the overall increase in the internal and external earth pressures, particularly at the soil outside the wall toe, may have larger physical relevance in terms of the behaviour of the walls and interpretation of the earth pressures mobilised. In addition, the maintained congruency between the columns of the sensing strips increases the confidence more generally in the data collected. While it is recognised averaging across the soil height would result in even smoother distributions, this would reduce the spatial resolution of the sensing sheets and can erroneously reduce peaks in the measured soil pressure distribution. Thus, for the remainder of the work the soil pressure distributions will be calibrated and corrected as shown in Figure 7.

Figure 8 illustrates the full procedure carried out on the swingup data from the test. As previously discussed, due to the installation of the walls before swing up, the distribution of stresses in Figure 8 is not reflective of the static pressures that are likely to develop in the field. Nevertheless, the distributions do appear physically reasonable. Both distributions show a general increase with depth, as one would expect as the vertical effective stress increases. Further, the soil on the outside of the wall shows larger stresses, as dictated by the condition of horizontal equilibrium. As the model is swung up it should be expected that the internal earth pressures tend to be active-like states while externally some amount of the passive limit is mobilised. Potentially, there will also be a change of mobilised earth pressure coefficient with g level due to the stress-dependent change of dilatancy. At $60 \mathrm{~g}$ near the external soil surface

$\approx 40 \mathrm{kPa}$ is mobilised both internally and externally. This stress implies KInt $\approx 0.35$ and KExt $\approx 2.08$, showing the soil has tended away from the at-rest conditions where $\mathrm{KO} \approx 0 \cdot 46$.

\section{Measuring Dynamic Earth Pressures}

Having established a technique to process earth pressure data from the centrifuge tests, the use of the pressure-sensitive sheets to measure the dynamic earth pressures generated around the retaining wall can be demonstrated. Following from Section 4.3 , the response during the first large earthquake motion will be considered. This was a tone burst with a prototype magnitude of $0.35 \mathrm{~g}$. This was preceded by a much smaller tone burst of $0.03 \mathrm{~g}$. Dynamic earth pressure distributions during early cycles will be interpreted with a view to assessing the consistency of the results produced. Prior to this however, a brief consideration of the dynamic response of the sheets and their practical use as part of a centrifuge dataset will be given. A detailed investigation of the dynamic response of the Tekscan sheets is presented by Dashti et al. (2012).

\section{Dynamic Response}

The Tekscan sheets were logged at a sampling rate of approximately $700 \mathrm{~Hz}$. While this is lower than the sampling rates typically used for instruments in dynamic tests (._6 kHz), it is still higher than the Nyquist frequency for most earthquake events at intermediate $\mathrm{g}$ levels. Taking earthquakes to have most of their energy between 1 and $3 \mathrm{~Hz}$, at $60 \mathrm{~g}$ this implies a Nyquist frequency of $360 \mathrm{~Hz}$. Sampling faster than this limits any higher frequency signals being aliased onto the measurements, be it from noise or structural vibrations.

Dashti et al. (2012) considered the dynamic performance of the Tekscan sheets in terms of the required sampling rate and the frequency response of the sheets. The sheets were found to attenuate 
signals at higher frequencies, which can be corrected using an experimentally derived transfer function. At $100 \mathrm{~Hz}$, Dashti et al. (2012) find the correction required to be of the order of 1.2. Due to differences in the set-up and system used, the correction was not applied to the results in this paper although the possible under predictions of peak stresses at large loading rates should be borne in mind.

A bigger consideration could be the temporal synchronisation of the data sets. Because the Tekscan sheets use proprietary logging software the need to synchronise the recorded earth pressures with other instruments in the centrifuge package can arise. Traditionally, this is done by way of peak matching techniques between time traces although some care must be taken when synchronising the earth pressures with other instruments. This is particularly pertinent when the sheets are installed vertically in the model, due to the increasing lag in the transmission of forces with height through the soil column. In the work presented here, the result of the double integral of thearth pressure distribution over the wall height was synchronised in time with strain gauges measuring the wall bending. It should also be noted that this ignores the effect of the small but finite multiplexing delays introduced by the Tekscan recording system. The veracity of this choice will be touched on in Section 5.2 .

Figure 9 illustrates the temporal variation of the lateral earth pressures in response to the applied tone burst. From Figure 9(a) a number of general points may be deduced. The traces plotted are taken from various depths along the inside and outside of the wall. As one would expect, larger lateral static pressures are mobilised with depth. The rate of increase is due to the linearly increasing vertical stress but also the mobilised earth pressure coefficient. The data from swing up, plotted in Figure 8, reminds us that this is not necessarily constant with depth. It is interesting to note that despite the symmetric acceleration input the oscillations in the soil pressures are biased towards the loading direction. However, the rate of change is seemingly not constant during the loading, occurring more rapidly as the driving force diminishes. This is particularly noticeable for the soil inside the wall toe. These observations, if physical, suggest that irrecoverable soil straining occurs and hence that stresses are 'locked-in' during the coseismic period. Madabhushi and Haigh (2018) consider this effect further. The dependence of this effect on depth is likely in part due to the differing magnitudes of the dynamic displacement along the flexible wall, as well as the natural increase of the soil stiffness with depth.

Figure 9 (b) zooms in on the variation with time and reveals interesting phenomena in terms of both the frequency and magnitude of the earth pressure oscillations and again the dependence this has on depth of the soil. For the stiff walls in Madabhushi and Haigh (2016b), the internal and external earth pressures were found to cycle in anti-phase, which was likened to the soil cycling between active and passive-like conditions. However, for the case of the lighter and more flexible walls presented in Figure 9(b) the internal and external pressure cycling is largely in phase. This is still possible as the dynamic active limit state is greater than the static one while the mobilised passive earth pressure coefficient can still increase up to the (lower) dynamic passive limit state. The influence of the wall stiffness and mass on the proportions of kinematic and inertial loads attracted by the system requires further study, but the useful insight gained into the phasing of the earth pressures from the tactile pressure sheets has been demonstrated.

The frequency of the oscillation appears larger than that of the input motion, especially for the internal pressure at the dredge level. Tricarico et al. (2016) and Madabhushi and Haigh (2016a) observed fluctuations in the tie force at double the driving frequency, and it is interesting to see this manifest in the earth pressures as well. 
More generally, the temporal variations of the earth pressures do appear rather coarser than typically seen with more traditional instrumentation. For the frequencies of variations within a cycle of the applied acceleration the dynamic response of the sheets may start to become a limiting factor. This suggests that interpretation of the peak and troughs of the earth pressure data may be more accurate than the intracyclical transitions.

\section{Interpretation of Results}

Figure 10 illustrates three example soil pressure distributions recorded at various points in the cycle of the tone burst, following the previously discussed processing techniques. Previous observations concluded that the average phase lag in the accelerometers at the wall height is approximately a quarter of a cycle (Madabhushi and Haigh, 2016a). Thus, time 1 and time 3 were chosen to illustrate the earth pressures at instants of close to maximum loading but in opposite directions, while time 2 illustrates a transitory time between them.

At time 1 very large earth pressures are mobilised over approximately the top $2 \mathrm{~m}$ of the external soil. The soil in this region is likely approaching a passive-like limit state. It is notable that the pressures rapidly decrease below $2 \mathrm{~m}$ depth. At time 3 there is a large decrease in the external lateral earth pressures where the previously observed passive-like state may have cycled to more active-like conditions. Simultaneously, inside the wall a noticeable increase develops across the middle section of the wall. Between these times the external soil pressures at time 2 fall reasonably within the states at time 1 and time 2, although this is less clear for the pressures inside the wall.

It is likely that larger pressures are mobilised where the wall movements are larger. For the flexible walls tested, the distributions in Figure 10 suggest that the greatest relative dynamic displacements occur in the middle portion of the wall. For a dual-row system with flexible walls with a tie rod at the wall heads is physically reasonable. More pertinently, it is evident that the distributions measured in Figure 10 may not readily be captured by simplistic triangular distributions that may be assumed in design calculations.

A difficulty with using the tactile pressure sensors in novel applications is how to establish the accuracy of the suggested pressure distributions. The use of additional instruments to see if the data forms a consistent set is one option. Figure 11 offers a comparison between the dynamic bending moment distribution as measured by the strain gauges on the right wall and the distribution implied by integrating the soil pressure distributions. This was carried out for the same three times plotted in Figure 10, although slight discrepancies between the gauges and the Tekscan are possible due to the different sampling rates.

The soil pressure distributions at these times were numerically integrated twice using the boundary conditions that the bending moment must be zero at the top and bottom of the wall. From Figure 11 it can be seen that a few salient features are correctly predicted, particularly at the instants time 1 and time 3. Generally, the recorded earth pressure distributions correctly capture the change in bending moment as the applied acceleration reverses. The tie rod is always in tension and thus positive bending moments are always generated at the wall heads. The rate and extent of the reversal of this bending moment depend on whether the wall is accelerating into or away from the external soil. Revisiting Figure 10, the large variation in the earth pressures generated outside the wall during the cycle is clearly responsible for this behaviour. The changing location of the peak bending moment between these instants is also correctly identified. 
Nevertheless, there are some discrepancies between the two distributions of bending moments in Figure 11. The intermediate time 2 exaggerates the instantaneous reversal of bending moment compared with the strain gauges. As previously mentioned, a lack of dynamic response, perhaps in registering the rapid unloading of earth pressures, could be responsible. Further, the gradient of bending moment at the wall heads is slightly different between the two methods. This suggests the tie force implied by the earth pressure distributions may be larger than reality. Overall, the match at these instants between the two measurement techniques is pleasing and combining Figures 10 and 11 facilitates greater insight into the problem being investigated.

\section{Conclusions}

The concept of two parallel rows of retaining walls used to withstand tsunami loading is an intriguing idea from both a structural and geotechnical viewpoint. Ensuring the performance of these structures under the preceding earthquake loading is important, and the use of a geotechnical centrifuge offers an accurate and cost-effective option to investigate the problem.

Traditional instrumentation such as strain gauges and load cells can be used to assess the structural performance, but the behaviour of the dual-row structure will ultimately depend on the soil pressures developed inside and outside the walls. The potential of tactile pressure sensors to elucidate the soil behaviour under dynamic loading was originally discussed in Madabhushi and Haigh (2016b) and is discussed further in this paper.

In agreement with previous work in this area, the need to carefully calibrate the sheets in order to improve the accuracy of the pressure measurements was highlighted. A method of accounting for the different sensitivity of the sensing elements while calibrating was utilised, which can use data collected from swing up without the need for additional centrifuge tests or models. The reduction in the data scatter was illustrated; however, the assumption of plane strain is necessary in order to justify the sensitivity adjustment. Further, the impact of the sensitivity adjustment correcting for both the natural variation in sensitivity of the sensing elements and the non-uniform contacts during swing up is considered. Significant grain rearrangement will thus reduce the appropriateness of sensitivity adjustment factors obtained from swing up. Nevertheless, for the tests presented even following several large shakes the scatter in earth pressure measurements was reduced using the calculated adjustments.

The relationship between the pressures around the wall registered by the sheets and the input acceleration was shown and the phase between the soil inside and outside the wall highlighted. The soil pressure distributions measured during the earthquake exhibited behaviour reminiscent of the active and passive states usually assumed but did not increase linearly with depth or respond symmetrically for positive and negative accelerations.

This paper presents a procedure for processing the data from tactile pressure-sensing sheets in order to better understand the results from a centrifuge test. In a simplified way, the results during the early cycles have been sense checked to improve confidence in the data. Overall, the use of tactile pressure sensors in this way offers unique insights into the soil behaviour and thus the dual-row system. 


\section{List of Figures}

1 Dual Row Retaining Wall Concept . . . . . . . . . . . . . . . . . . . . . . . . 10

2 Simplified Centrifuge Model Schematic . . . . . . . . . . . . . . . . . . . . . . . . . 11

3 Illustrative Raw Output from Tekscan Sensors . . . . . . . . . . . . . . . . . . . . 12

4 Procedure for obtaining Earth Pressures from Tekscan data . . . . . . . . . . . . . . . 13

5 Tekscan Calibration - cycling input pressure . . . . . . . . . . . . . . . . 14

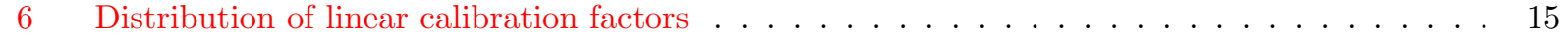

$7 \quad$ Correcting for the sensing element sensitivity individually $\ldots \ldots \ldots \ldots \ldots$

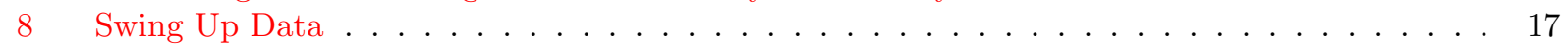

9 Dynamic Variation of soil pressures . . . . . . . . . . . . . . . . . . . . . . 18

10 Soil pressures measured during earthquake loading . . . . . . . . . . . . . . . . . 19

11 Comparing bending moments from strain gauges and predictions inferred from Tekscan . . . 20 


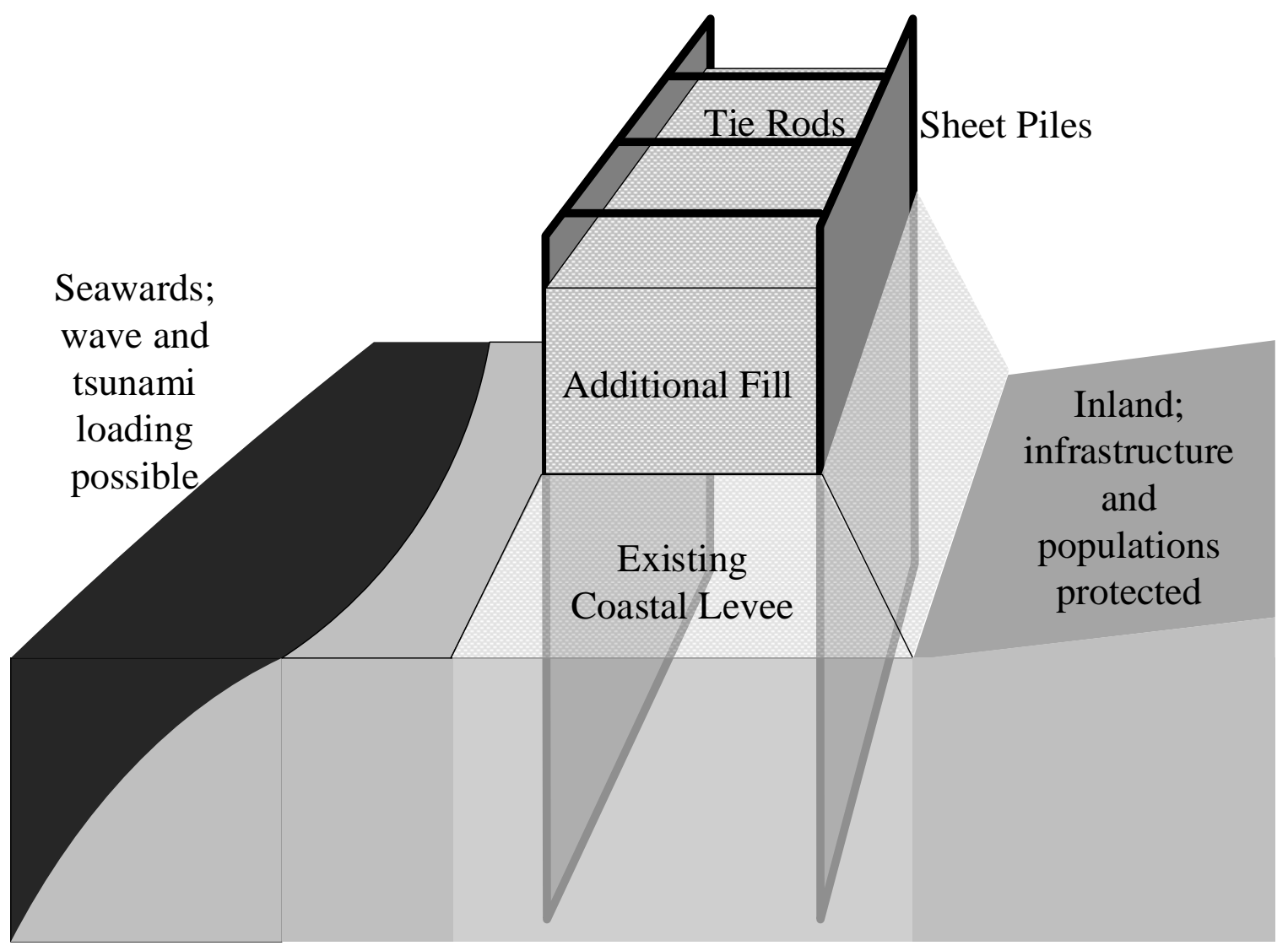

Figure 1: Dual Row Retaining Wall Concept 


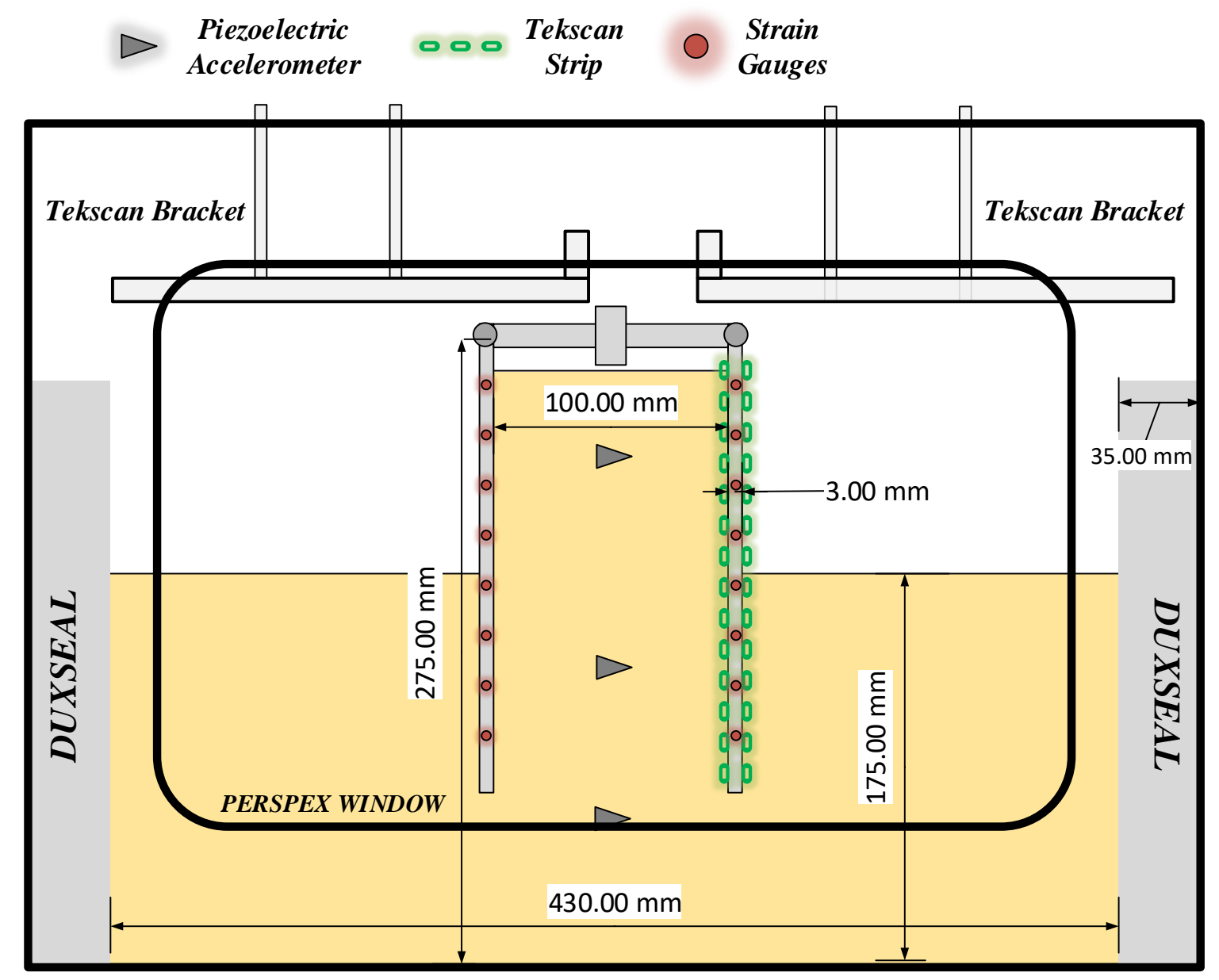

Figure 2: Simplified Centrifuge Model Schematic 


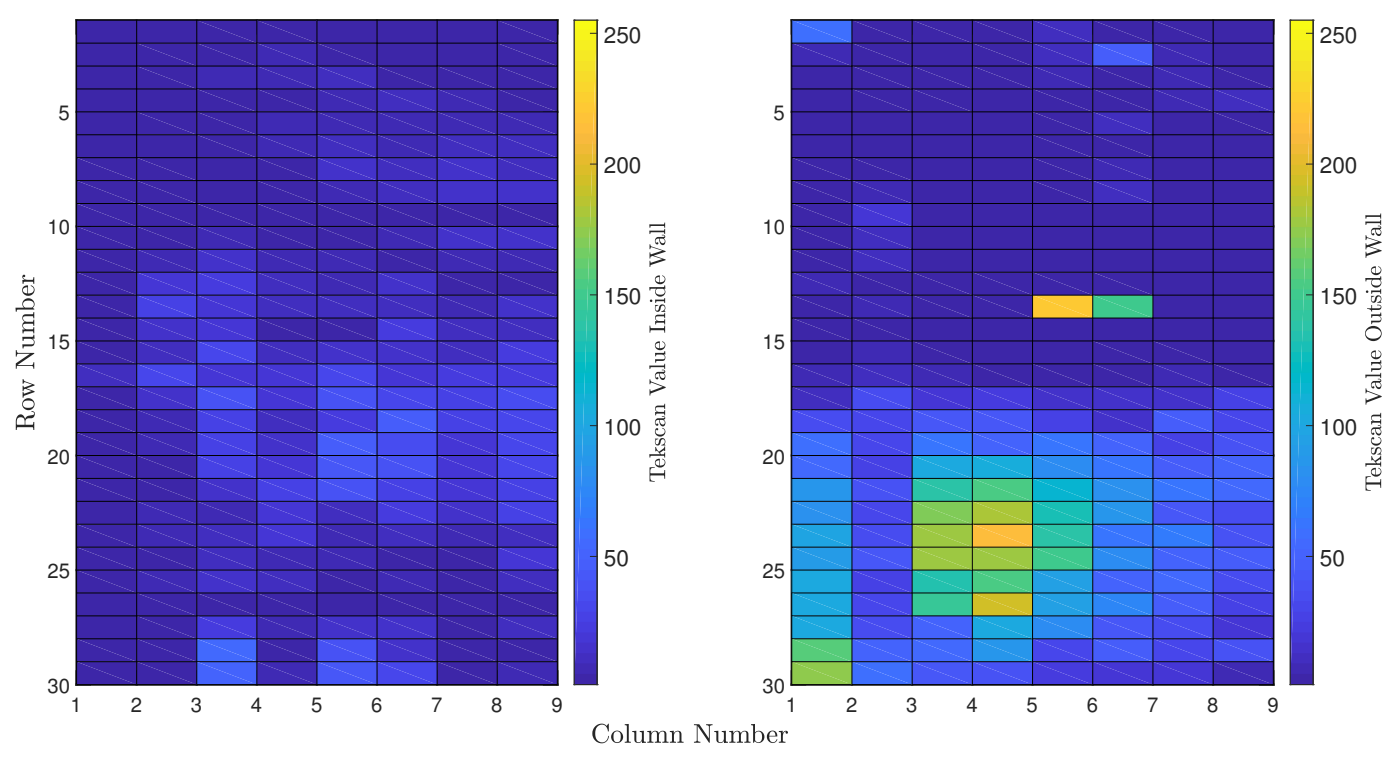

Figure 3: Illustrative Raw Output from Tekscan Sensors 


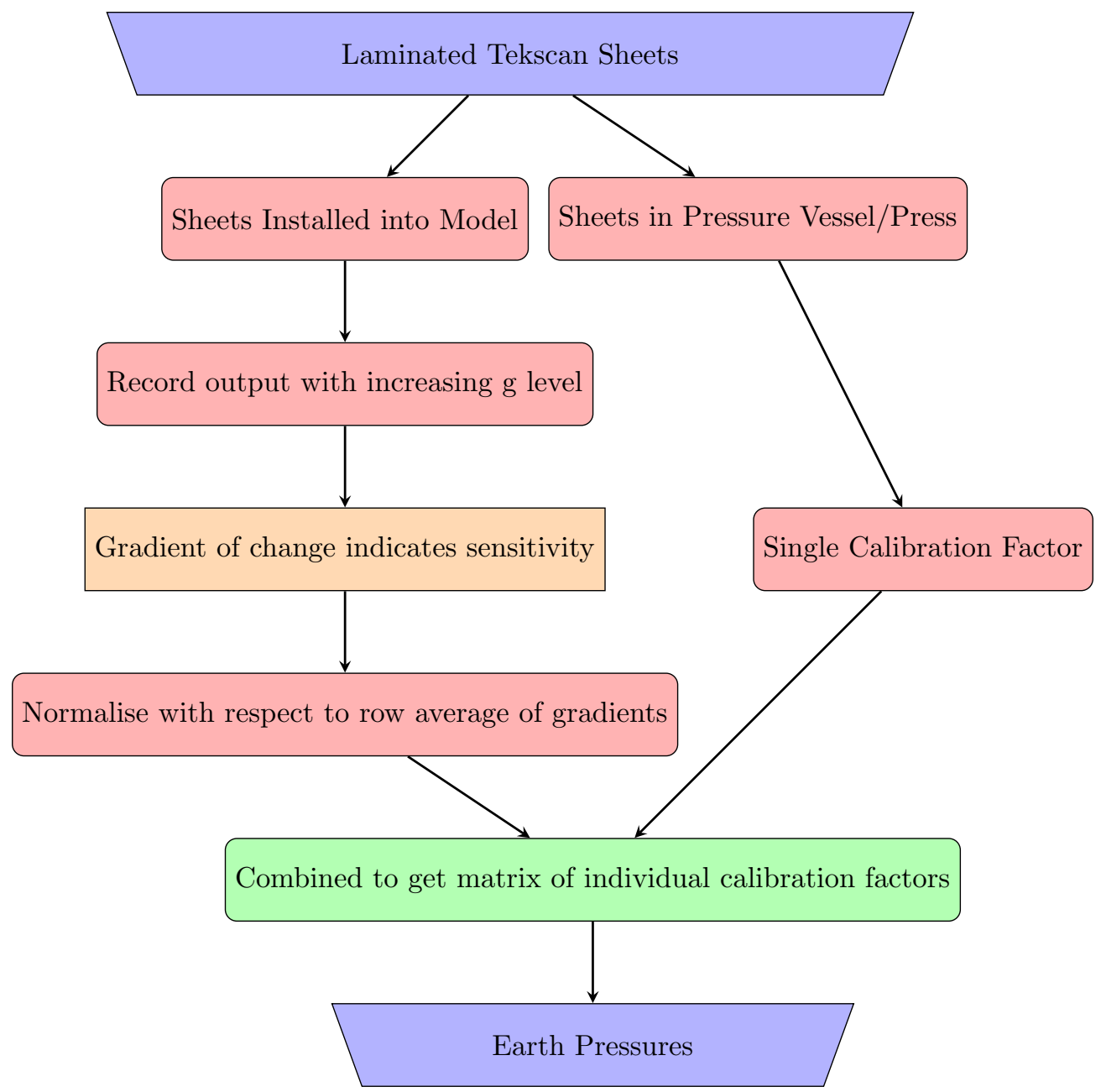

Figure 4: Procedure for obtaining Earth Pressures from Tekscan data 

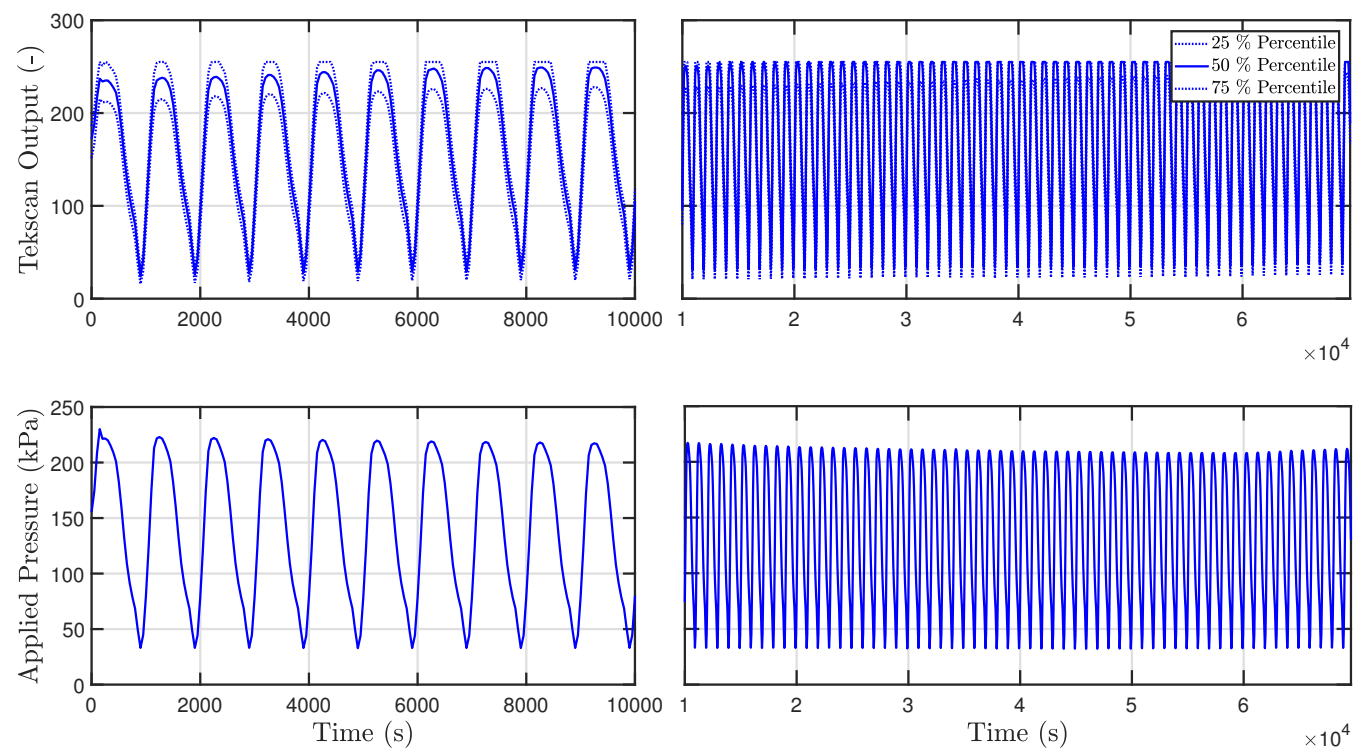

Figure 5: Tekscan Calibration - cycling input pressure 

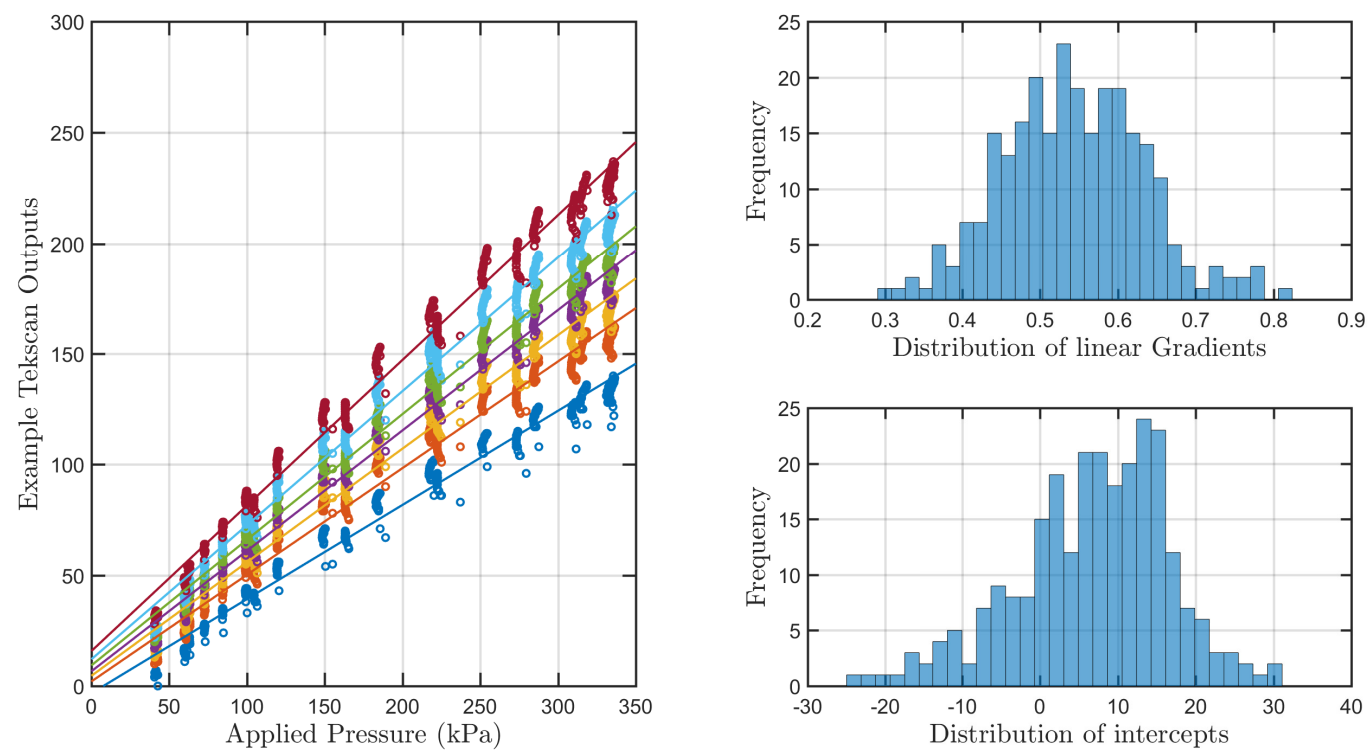

(a) Direct Contact with sheet
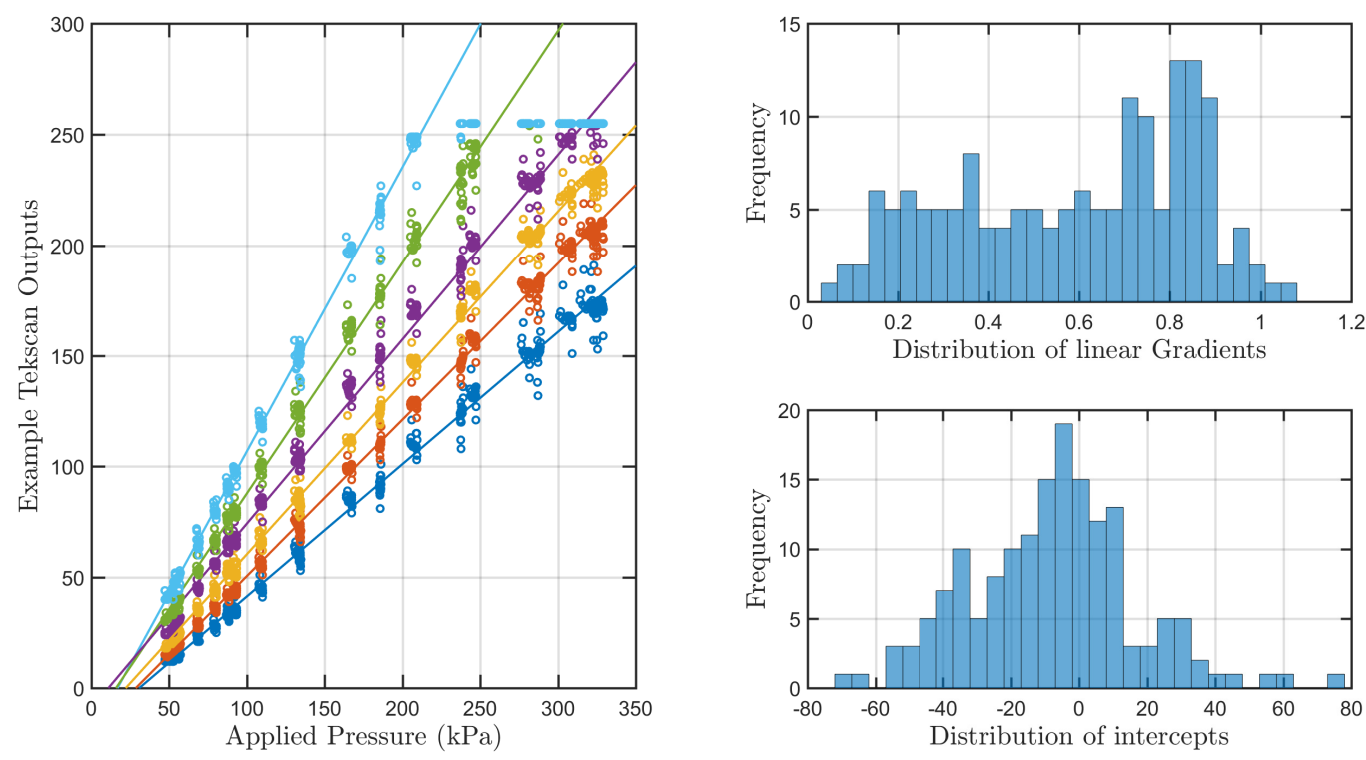

(b) Pressure applied through Sand Layer

Figure 6: Distribution of linear calibration factors 

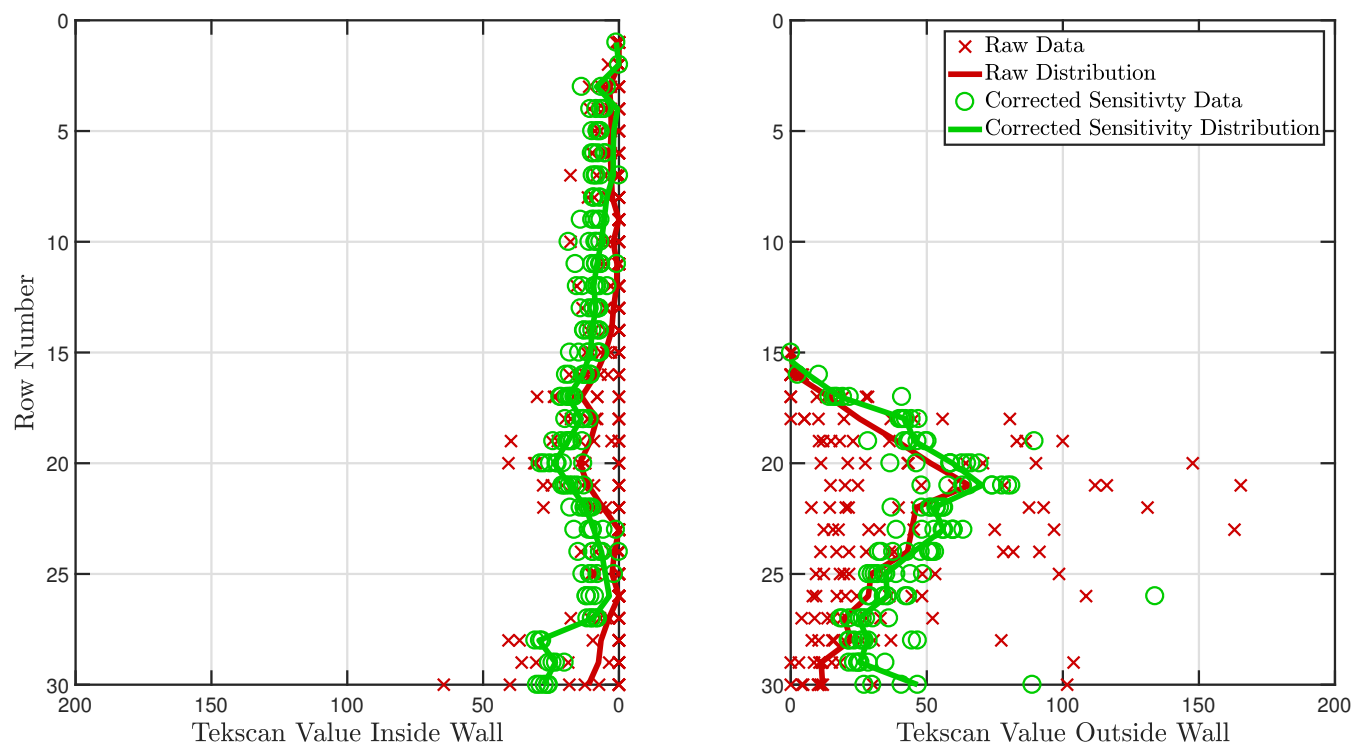

(a) Sensitivity Correction, following one small shaking motion
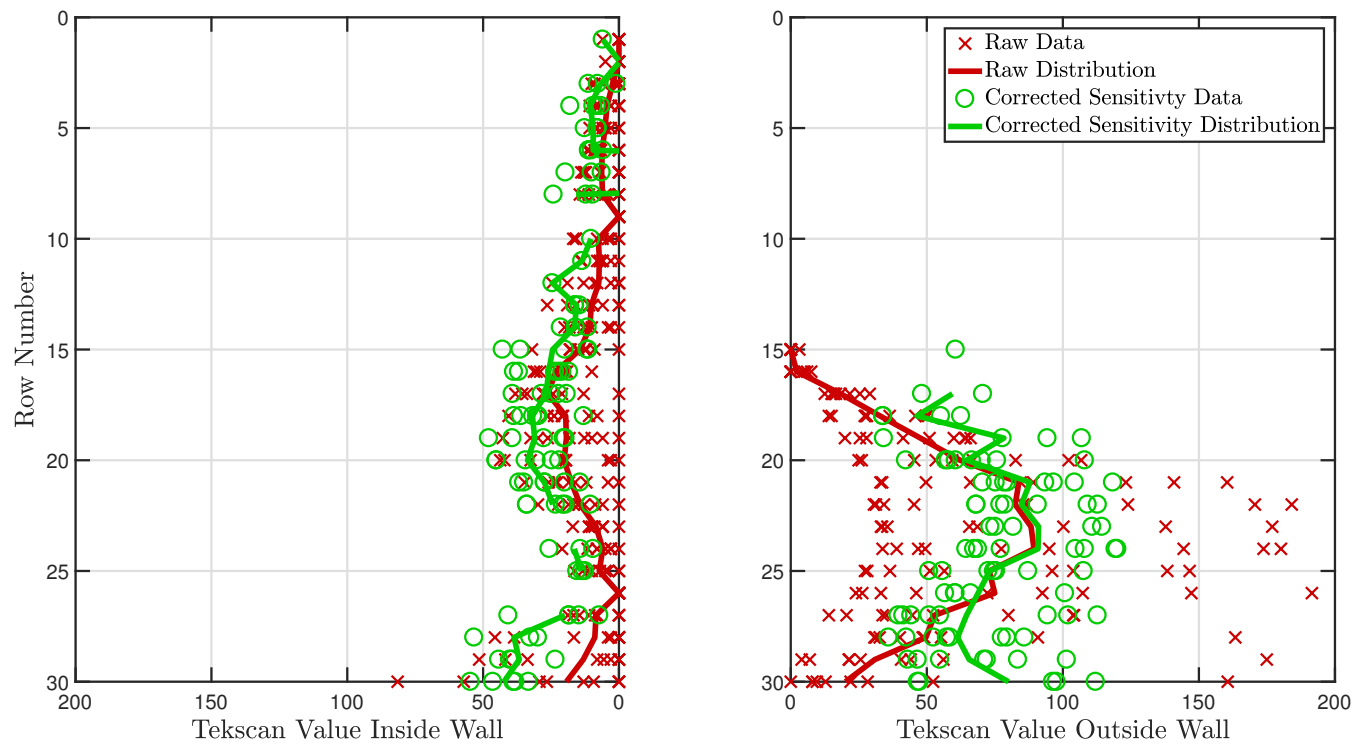

(b) Sensitivity Correction, following several large shaking motions

Figure 7: Correcting for the sensing element sensitivity individually 

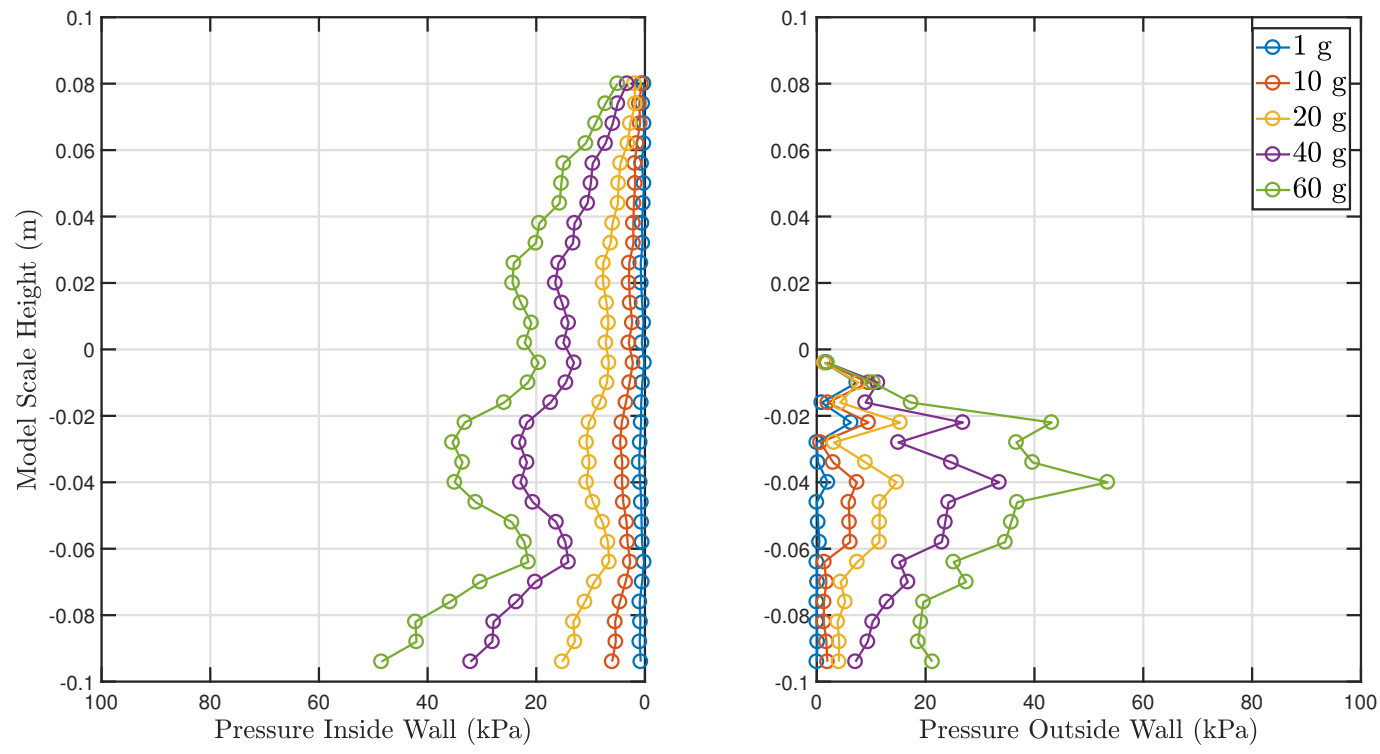

Figure 8: Inferred Soil Pressures During Swing Up 

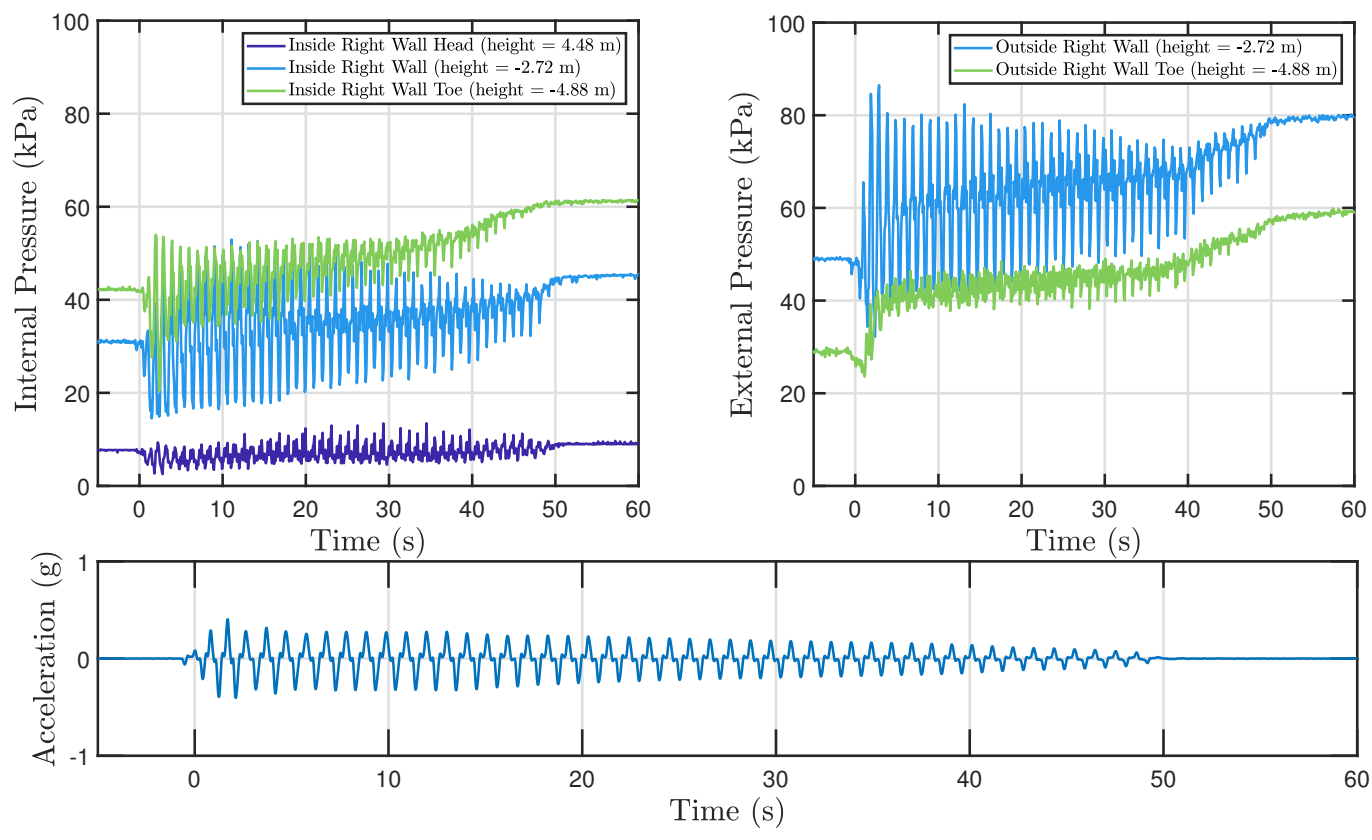

(a) Cyclic Variation in earth pressures
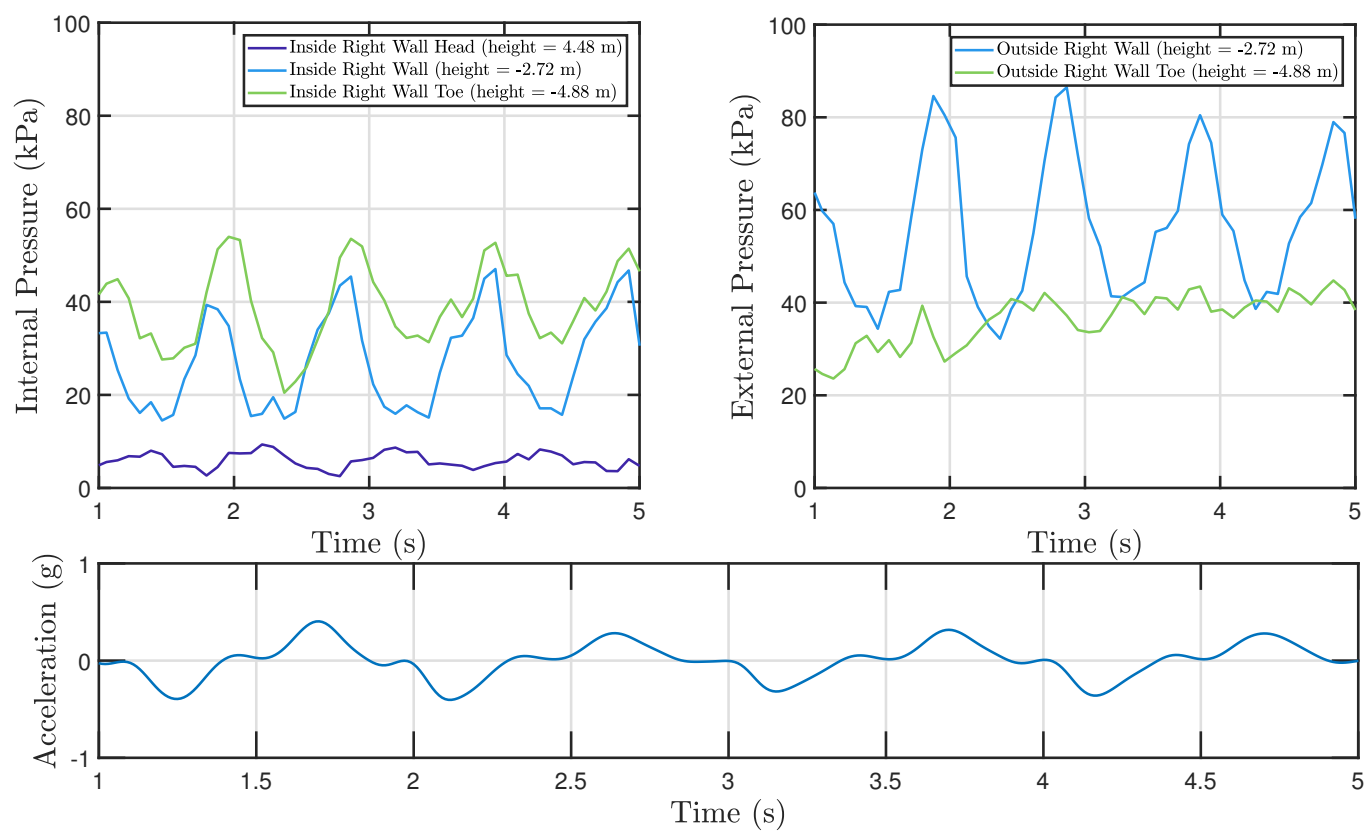

(b) Zoomed Cyclic Variation in earth pressures

Figure 9: Dynamic Variation of soil pressures 

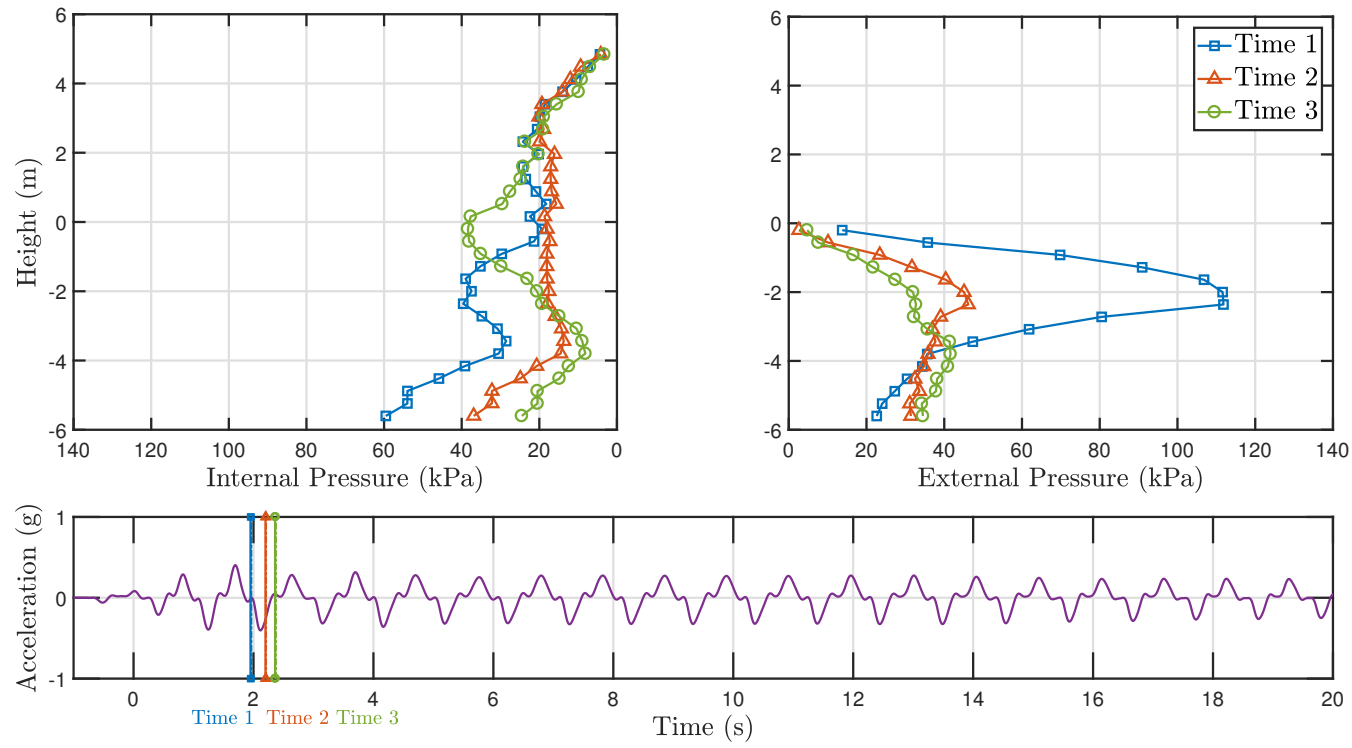

Figure 10: Soil pressures measured during earthquake loading 

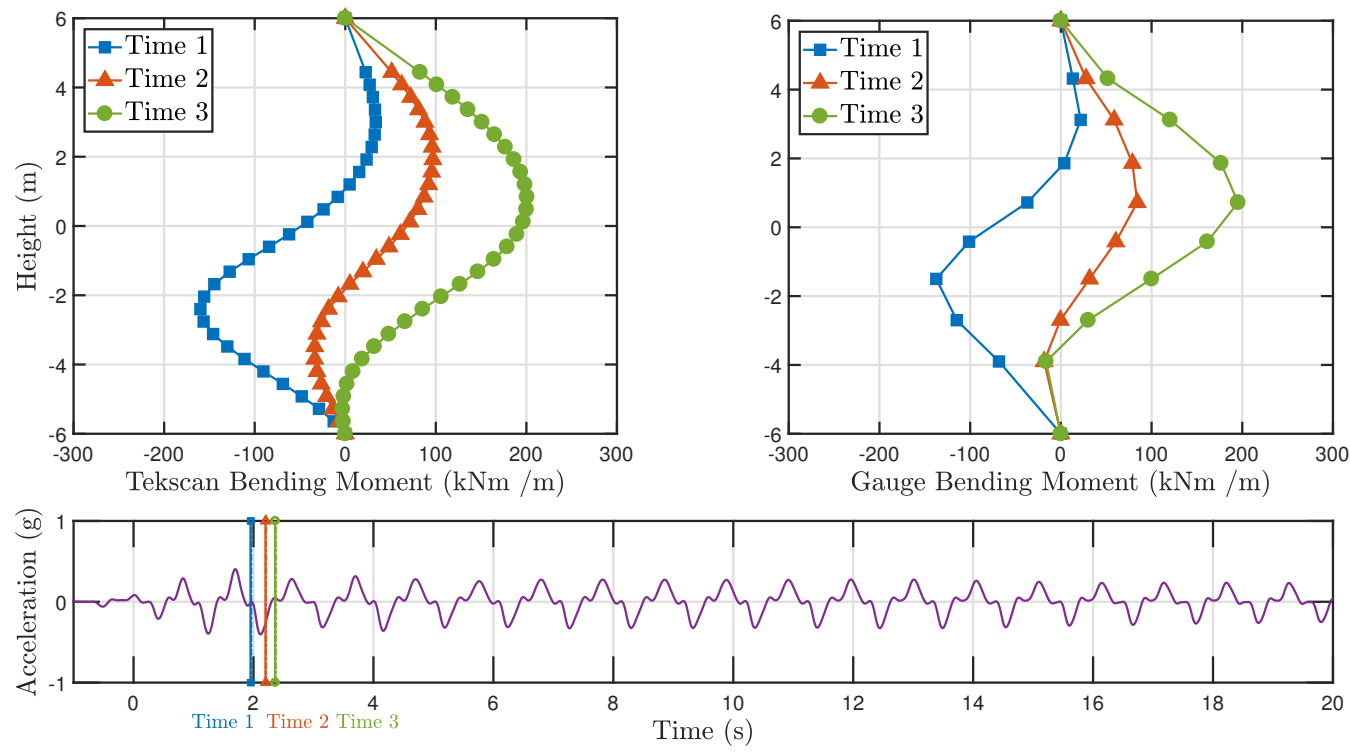

Figure 11: Comparing bending moments from strain gauges and predictions inferred from Tekscan 


\section{List of Tables}

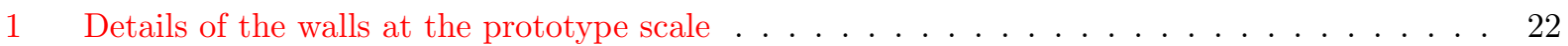




\begin{tabular}{ccc}
\hline Variable & Value & Unit \\
\hline Total wall height & 12 & $\mathrm{~m}$ \\
Embedment depth & 6 & $\mathrm{~m}$ \\
Wall thickness & 0.18 & $\mathrm{~m}$ \\
Bending stiffness & 34 & $\mathrm{MNm}^{2} / \mathrm{m}$ \\
Tie length & 6 & $\mathrm{~m}$ \\
\hline
\end{tabular}

Table 1: Details of the walls at the prototype scale 